\title{
Implementation of a Clinical Decision Support Systems- Based Neonatal Monitoring System Framework
}

\author{
Sobowale A. A $\mathrm{A}^{1}$, Olaniyan O. $\mathrm{M}^{2} *$, Adetan. $\mathrm{O}^{3}$, Adanigbo. $\mathrm{O}^{4}$ \\ Esan. A $^{5}$, Olusesi. A.T ${ }^{6}$, Wahab. W.B ${ }^{7}$, Adewumi. O. A $^{8}$ \\ Department of Computer Engineering, Federal University Oye-Ekiti, Nigeria ${ }^{1,2,4,5}$ \\ Department of Electrical and Electronic Engineering, Ekiti State University Ado-Ekiti, Nigeria ${ }^{3}$ \\ Department of Electrical, Electronics \& Computer Engineering, Bells University of Technology Ota, Nigeria ${ }^{6}$ \\ Department of Computer Engineering, Ladoke Akintola University of Technology Ogbomoso, Nigeria ${ }^{7}$ \\ Department of Computer Science and Information Technology, Bells University of Technology Ota, Nigeria ${ }^{8}$
}

\begin{abstract}
A Clinical Decision Support-based information systems to monitor the vital signs of the neonate's conditions in prematurely born babies placed in infant incubators of Neonatal Intensive Care Unit (NICU) is developed in this work. A DMS was developed consisting of a supervisory microcomputer and sensitive sensors for measuring the vital signs. The Conventional Monitoring System (CMS) was used simultaneously with the DMS to collect the vital sign readings of thirty (30) neonates, over a period of one week. Fuzzy Inference System CDSS (FIS-CDSS) was developed for the three inputs: Temperature, Heart rate and Respiration rate (THR) based on their membership functions' value (low, medium, high) and twenty-seven (27) IF-THEN fuzzy rules using fuzzy logic toolbox in Matrix Laboratory 8.1 (R2014a). The FIS-CDSS maps the THR to an output status (Normal, Abnormal and Critical). The performance of the FISCDSS was evaluated using confusion matrix. The results showed that the system yielded sensitivity ranges of $90-100,80-89,70$ 79, 60 - 69 and $50-59 \%$ for five, eleven, seven, six and one neonates, respectively with an average sensitivity of $77.92 \%$. The specificity of the system ranged from 5.00 to $66.67 \%$ with an associated average specificity of $35.10 \%$. The accuracy of the FIS-CDSS ranged from 70 to 100,60 to 69,50 to 59 and 0 to $49 \%$ for nine, nine, eight and four neonates, respectively with an average accuracy of $60.94 \%$. The developed system provides adequate and accurate information for on-the-spot assessment of neonates for decision making that improves the mortality rate and recovery period of neonates.
\end{abstract}

\section{General Terms: Neonatal Monitoring}

Keywords-Clinical Decision Supports Systems (CDSS); Fuzzy Inference System (FIS); Neonatal Intensive Care Unit (NICU); vital signs; neonates

\section{INTRODUCTION}

Decision Support Systems (DSS) are increasing in coverage of different sections of life which includes academic, engineering, business, military and medicine [1]. Any automated program that helps specialists in settling on clinical choice is categorized as Clinical Decision Support Systems (CDSS) [2]. CDSS provide clinicians, staff, patients and other individuals with knowledge and person-specific information, wisely separated and displayed at proper times, to upgrade wellbeing, medicinal services and reduce medical errors [3][4][5]. CDSS does not decide; It just gives direction to provide current and pertinent knowledge to clinicians to aid patient care at the exact time of care delivery [6][7] It is a major technology application to make the right decision at the right time which aids in building an intelligent system for monitoring neonatal vital parameters [8]. The CDS Systems are computer-based information systems used to integrate clinical and patient information to provide support for decision-making in patient care. A category of such patients are the prematurely born babies, which are placed in infant incubators of Neonatal Intensive Care Unit (NICU) for continuous monitoring of their body vital signs (temperature, heart rate and respiration). [19].

Neonates born before thirty-seven (37) weeks gestation are considered premature and are usually in a fragile condition and may be at risk of complications, such babies therefore require special monitoring and intensive care involving treatment in an incubator at an NICU [9][10][11]. Neonatal monitoring refers to the monitoring of vital physiological parameters of premature infants [12]. The survival rate of premature infants is dependent on the continuous monitoring of vital signs; this provides a lot of information about a baby's state of health [18].

In the last decades the advances in sensor technologies and wireless communications technologies have resulted in the possibility of developing intelligent systems for monitoring neonatal vital parameters [13]. Technology therefore provides easy data collection from the neonates monitoring system and aids the neonatologists' in taking appropriate decision.

However, the quality of neonatal care provided by Nigerian hospitals is not uniform and mostly manual, which creates difficulty of interpretation for inexperienced staff [14][15][16] More so, despite the impact of CDSS applications in various sectors of the health system, its application to monitoring of vital signs of preterm babies in the NICU is limited [5], [17].

This paper therefore developed a CDSS that can be used to efficiently monitor the neonate's condition in the incubators of NICU. The paper has five (5) sections in all. Section I is the introduction to the work. Section II gives the architectural framework of CDS systems. The methodology adopted in this work is discussed under Section III. Results obtained in this work are discussed under Section IV while the conclusion is given under Section VI.

*Corresponding Author 


\section{THE CDSS-BASED ARCHITECURAL FRAMEWORK}

The CDSS architectural framework is made up of three components (knowledge base, inference engine and interface) as shown in Fig. 1. This is made up of a set of functional and informational units. The functional unit is divided into the reasoning engine and the connection component. The informational unit comprises the data source and the knowledge base. The knowledge base consists of decision rules, low, medium and high boundary values, diagnosis terms, and clinical recommendation contents. The reasoning engine takes the readings of the vital signs as its data source. After the execution of the decision rules on the data source, the reasoning engine generates the output result, which is displayed on the monitor of the CDSS system and printed from the CDS located at the nursing stand. The clinicians take informed, on the spot decision based on the printed results. This enhances decision making and general performance as the manual routine checks by the nurses is no more the only basis of attending to neonates.

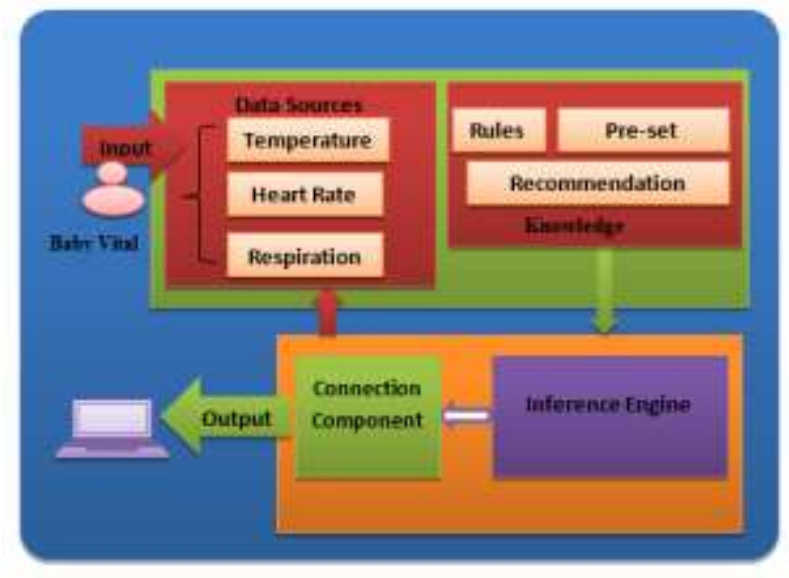

Fig. 1. The CDSS Architecture of the Developed Monitoring System.

\section{Methodology}

The Fuzzy Inference System (FIS) was developed using MATLAB R2014a to implement the CDSS architectural framework. The FIS uses fuzzy logic to map the vital signals Temperature, Heart rate and Respiration rate (THR) to a status (Normal, Abnormal and Critical). The output is used to decide on the appropriate treatment for a particular preterm. The FIS decisions are made by the use of membership function and IfThen rules. FIS performs fuzzification on the inputs and defuzzification of the result of fuzzy logic rule to determine the output. Aggregation is used to combine the output of all the rules into a single fuzzy set. The developed FIS takes the vital signs as the inputs and gives "Normal", "Abnormal" or "Critical" as the output. It also consists of the membership functions (MF), antecedents (or premise), consequents (conclusion), weight and connective. A membership function defines the degree to which the value of a vital sign falls within a boundary (or degree of membership). Antecedents are the MF values of the inputs while the consequents are the MF values of the output. A weight determines the level of importance of a rule relative to the others, and the maximum weight a rule can take is 1 . A connective takes either "AND" or "OR". The connective "AND" implies that the values of two antecedents determine the consequents while the connective "OR" implies that any of the antecedents can determine the consequents. The Graphics User Interface (GUI) of the developed FIS is shown in Fig. 2.

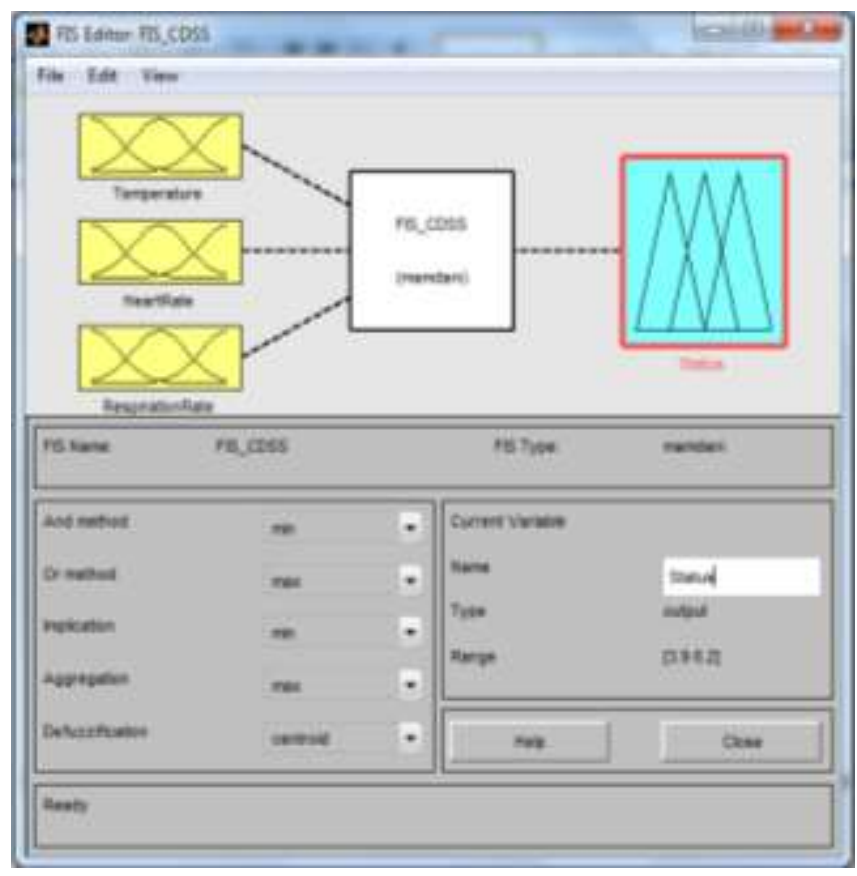

Fig. 2. GUI of the Developed Fuzzy Inference System in MATLAB Environment.

\section{A. Design of the Membership Function}

Three linguistic terms (Low, Medium and High) were used to define the membership function of each of the input variables Temperature, Heart Rate and Respiration rate (THR).

Similarly, three linguistic values Normal (N), Abnormal (A) and Critical (C) were used to define the membership function of the Status or Output (Out) of the inference engine. The value range of the vital signs readings used in the Children Intensive Care Unit (CICU) of Ladoke Akintola Teaching Hospital (LAUTECH) Osogbo, were used to set the range used in the FIS and were classified as Low for readings below the normal range, medium for normal range and high for readings above the normal range, this is discussed below.

Temperature: The normal range for Temperature is 36.5$37.5^{\circ} \mathrm{C}$; if the input temperature value is more than this range then its MF is High, and if it is below this range then its MF is Low. The classification of Temperature is presented in Table I(A). The MF for the fuzzy set for Temperature (Tmp) is defined as:

$$
\begin{aligned}
& \text { Low } \left.(T m p)=\left\{\begin{array}{cc}
\frac{1}{38-T m p} & T m p \leq 32.5 \\
1 & 32.5<T m p<36.5
\end{array}\right\} 1 a\right) \\
& \left.\operatorname{Medium}(T m p)=\left\{\begin{array}{cc}
\frac{T m p-32.5}{2} & 35 \leq T m p<37.5 \\
1 & T m p=37 \\
\frac{38-T m p}{1} & 37<T m p<38
\end{array}\right\} 1 b\right)
\end{aligned}
$$


$\operatorname{High}(\operatorname{Tmp})=\left\{\begin{array}{cl}\frac{T m p-37.5}{2} & 37.5 \leq \operatorname{Tmp}<39.5 \\ 1 & T m p \geq 39.5\end{array}\right\}$

Heart Rate: The normal range for Heart Rate (Hr) is 130$160 \mathrm{bpm}$; if the input heartbeat rate value is more than this range then its MF is High, and if it is below this range then its MF is Low. The classification of Heart Rate is presented in Table I(B). The MF for the fuzzy set for heart rate is:

Low $\left.\left(H_{Y}\right)=\left\{\begin{array}{cc}1 & H_{F}<125 \\ \frac{132-H_{Y}}{7} & 125<H_{Y}<132\end{array}\right\} 2 a\right)$

Medium $\left.\left(H_{r}\right)=\left\{\begin{array}{cc}\frac{H_{y}-128}{17} & 128 \leq H_{r}<145 \\ 1 & H_{r}=145 \\ \frac{162-H_{r}}{17} & 145<H_{r}<162\end{array}\right\} 2 b\right)$

$\operatorname{High}\left(H_{y}\right)=\left\{\begin{array}{cc}\frac{H_{y}-158}{12} & 158 \leq H_{y}<170 \\ 1 & H_{Y} \geq 170\end{array}\right\}$

Respiration Rate: The normal range for Respiration Rate $(\mathrm{Rr})$ is $40-60 \mathrm{~cm}$; if the input heartbeat rate value is more than this range then its MF is High, and if it is below this range then its MF is Low. The classification of Heart Rate is presented in Table $\mathrm{I}(\mathrm{C})$. The MF for the fuzzy set for respiration is:

Low $\left.\left(R_{y}\right)=\left\{\begin{array}{cc}1 & R_{y} \leq 35 \\ \frac{42-R_{r}}{7} & 35<R_{r}<42\end{array}\right\} 3 a\right)$

Medium $\left.\left(R_{\gamma}\right)=\left\{\begin{array}{cc}\frac{R_{r}-38}{12} & 38 \leq R_{y}<50 \\ 1 & R_{y}=50 \\ \frac{62-R_{r}}{12} & 50<R_{r}<62\end{array}\right\} 3 b\right)$

$\operatorname{High}\left(R_{y}\right)=\left\{\begin{array}{cc}\frac{R_{y}-60}{10} & 60 \leq R_{y}<70 \\ 1 & R_{y} \geq 70\end{array}\right\}$

Status: This is the output variable of the FIS. The normal range for Status (Out) is 4-6; if the output value is more than this range then its MF is Critical, and if it is below this range then its MF is Abnormal. The classification of Status is presented in Table I(D).

Abnormal (Out) $\left.=\left\{\begin{array}{cc}1 & \text { Out } \leq 3.5 \\ \frac{4-\text { Out }}{0.5} & 0.5<0 \text {.ut }<5\end{array}\right\} 4 a\right)$

Normal (Out) $\left.=\left\{\begin{array}{cc}\frac{\text { Out }-3.8}{1.2} & 3.8 \leq \text { Out }<5 \\ \frac{1}{1.2-\text { Out }} & \text { Out }=5 \\ 1.2 & 5<\text { Out }<6.2\end{array}\right\} 4 b\right)$

Critical (Out) $=\left\{\begin{array}{cc}\frac{0 u t-6}{0.2} & 6 \leq 0 \text { ut }<6.2 \\ 1 & \text { Out } \geq 6.2\end{array}\right\}$
The MF plots for Temperature, Respiration rate, Heart rate and Status are shown in Fig. 3 to Fig. 6.

TABLE I. A: ClASSIFICATION OF TEMPERATURE

\begin{tabular}{|l|l|l|}
\hline Vital Sign & Range & Linguistic Term \\
\hline Temperature & $<36.5$ & Low \\
\hline & $36.5-37.5$ & Medium \\
\hline & $>37.5$ & High \\
\hline
\end{tabular}

TABLE I (B): ClassificATION OF HEART RATE

\begin{tabular}{|l|l|l|}
\hline Vital Sign & Range & Linguistic Term \\
\hline Heart Rate & $<130$ & Low \\
\hline & $130-160$ & Medium \\
\hline & $>160$ & High \\
\hline
\end{tabular}

TABLE I (C): ClassificATION OF RESPIRATION RATE

\begin{tabular}{|l|l|l|}
\hline Vital Sign & Range & Linguistic Term \\
\hline Respiration Rate & $<40$ & Low \\
\hline & $40-60$ & Medium \\
\hline & $>60$ & High \\
\hline
\end{tabular}

TABLE 1D: ClassificATION OF STATUS

\begin{tabular}{|l|l|l|}
\hline Output & Range & Linguistic Term \\
\hline Status & $<4$ & Abnormal \\
\hline & $4-6$ & Normal \\
\hline & $>6$ & Critical \\
\hline
\end{tabular}

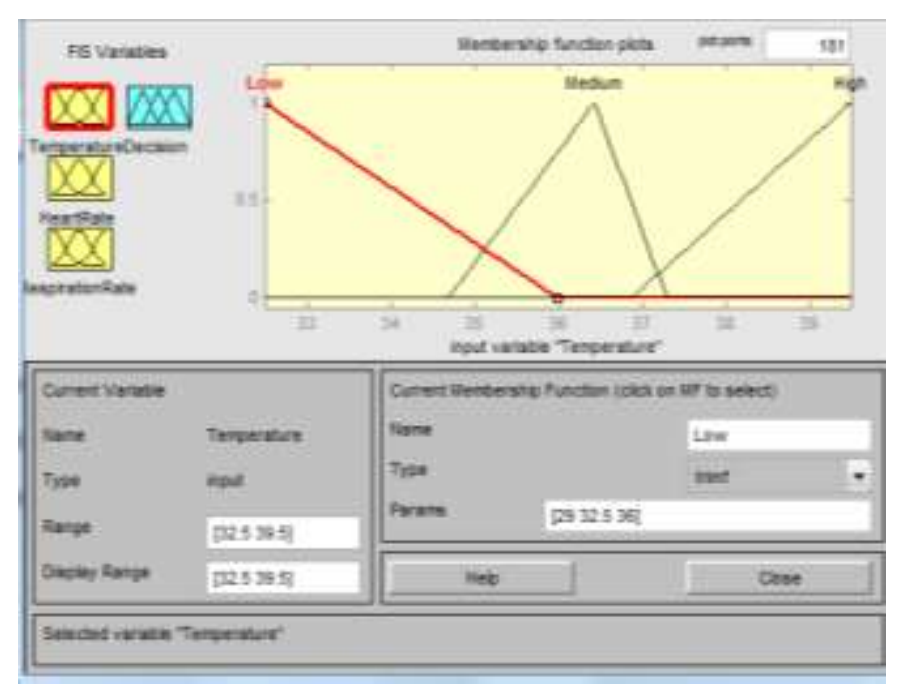

Fig. 3. Membership Functions for Temperature. 


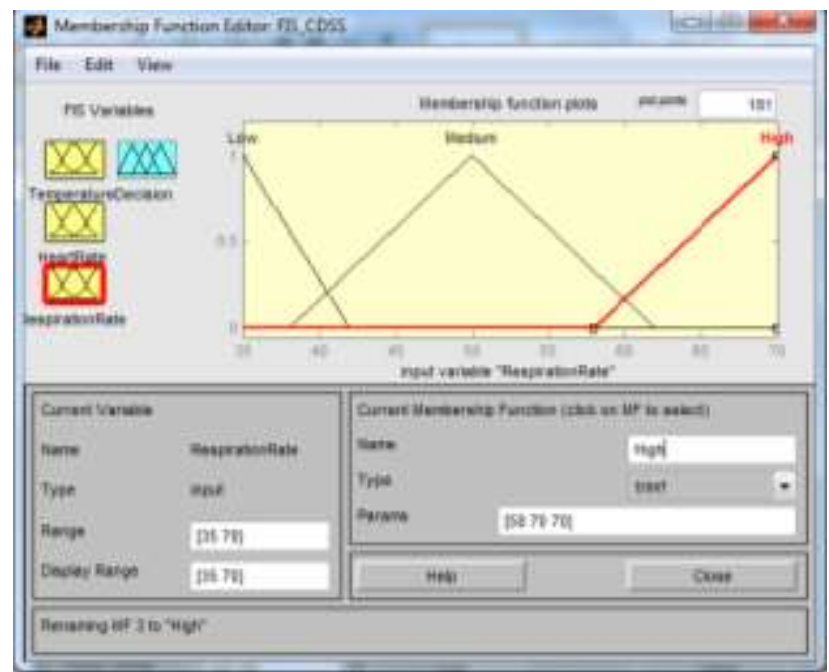

Fig. 4. Membership Functions for Respiration Rate.

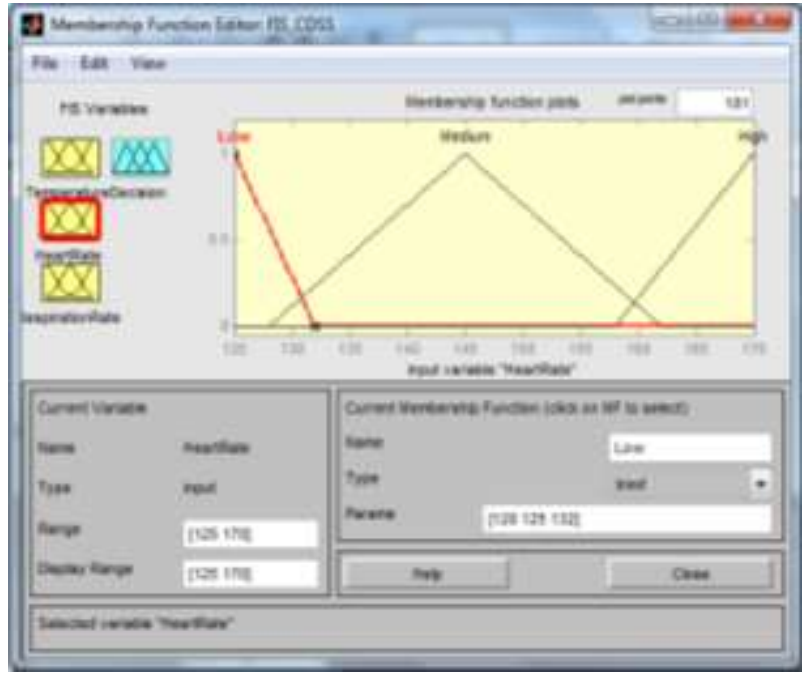

Fig. 5. Membership Functions for Heart Rate.

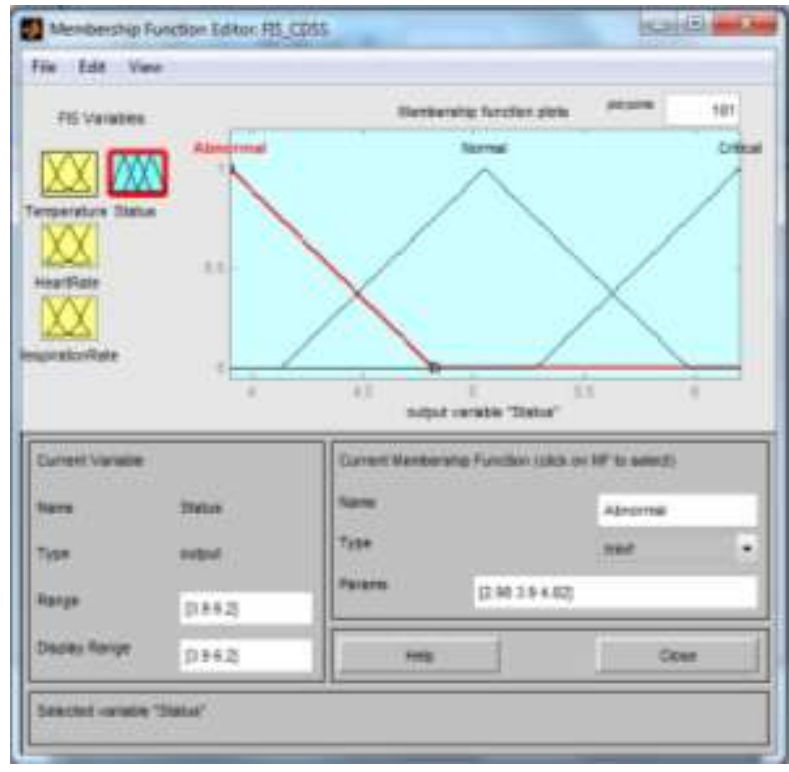

Fig. 6. Membership Functions for Status.

\section{RESULTS AND DISCUSSION}

An interactive Graphic User Interface (GUI) application was developed using MATLAB R2014a as the frontend and MYSQL 5.1 as the backend to implement the CDSS architectural framework. The developed system named Fuzzy Inference System Clinical Decision Support System (FISCDSS) was copied in a folder into the Clinical Database Server (CDS) with a Matlab file (FIS-CDSS_gui.m); the CDS contains database of the vital signs readings collected from the measuring sensors attached to each neonate. The FIS-CDSS GUI window (Fig. 7) appeared as the filename was executed. The vital signs (Temperature, Heart rate and Respiration) readings from the DMS were loaded into the developed FISCDSS as shown in Fig. 8. The loaded readings were run through the FIS-CDSS for classification as shown in Fig. 9 and Fig. 10. The CDSS_FIS classified the status of the baby (developed system prediction) as Normal, Abnormal or Critical based on the readings and the fuzzy logic rules in the knowledge base of the system; this is shown in Fig. 11, the developed system's prediction can be saved into the CDS as shown in Fig. 12.

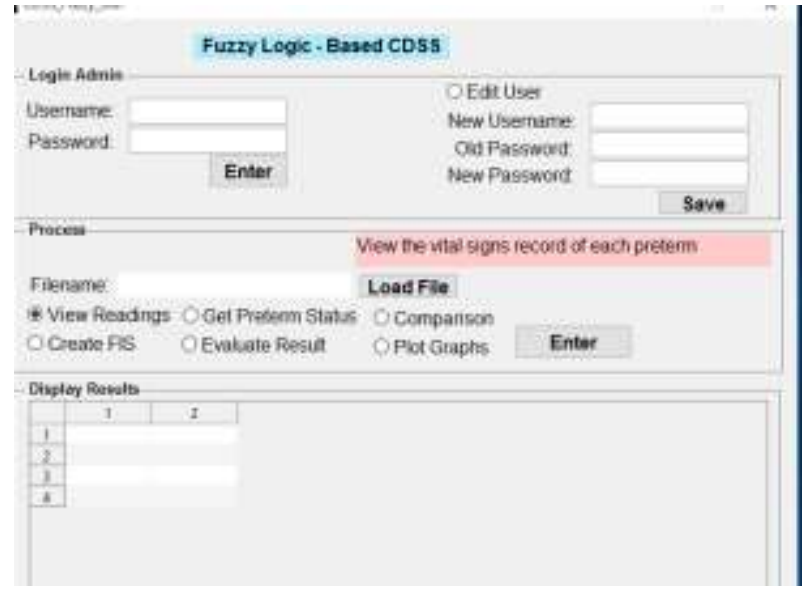

Fig. 7. The Developed FIS-CDSS Window.

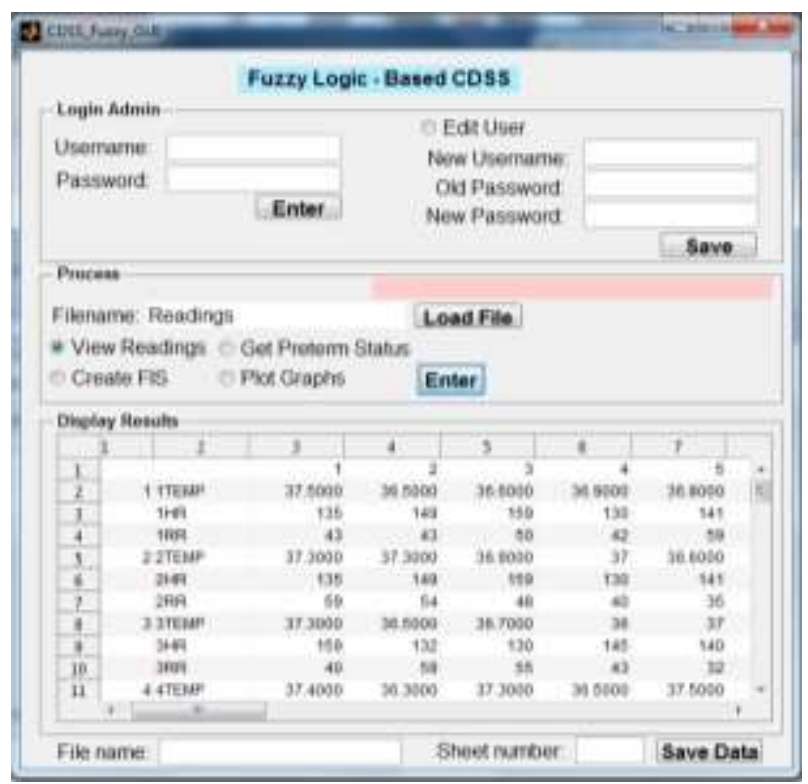

Fig. 8. Viewing of the Recorded Vital Signs on the GUI. 


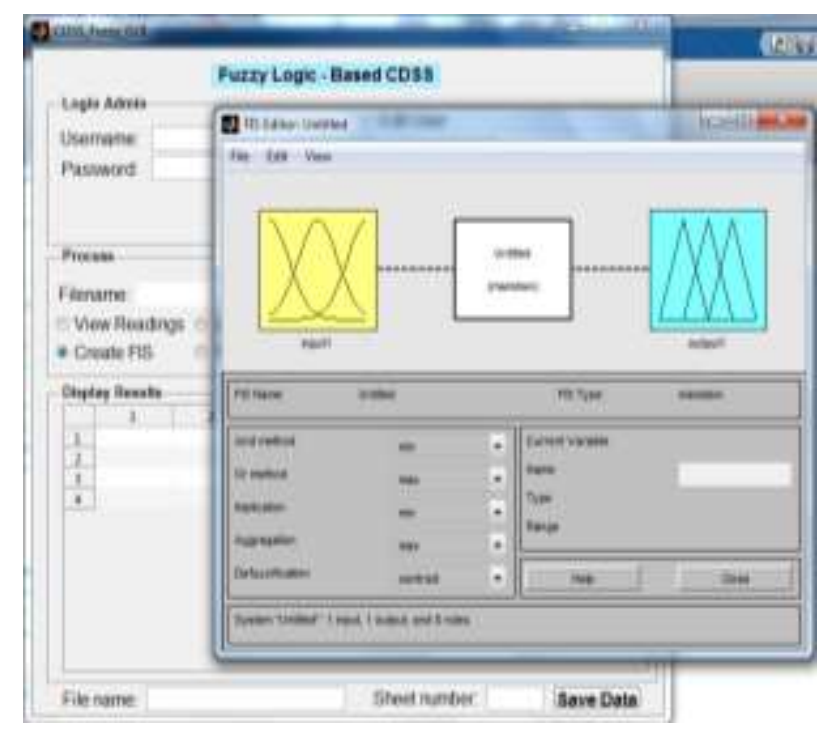

Fig. 9. Creation of a Fuzzy Inference System (FIS) Model for the CDSS.

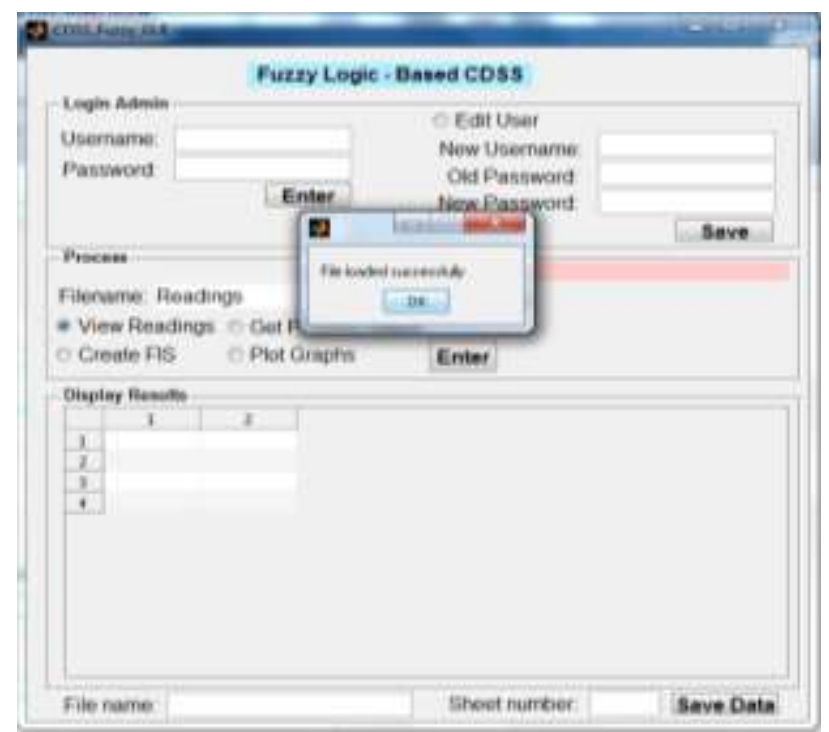

Fig. 10. Acquisition of the Recorded Vital Signs for the FIS Classification.

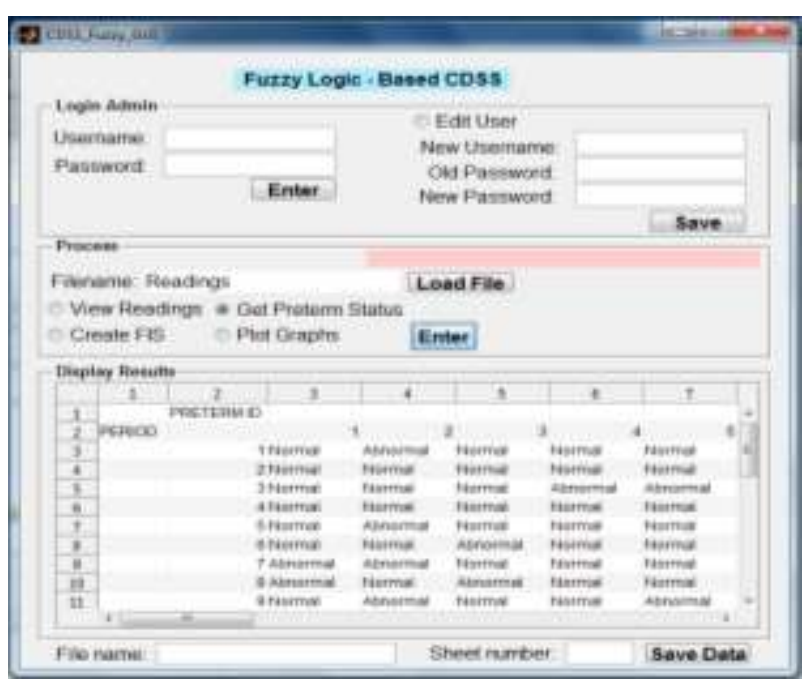

Fig. 11. Display of the FIS Classification Results.

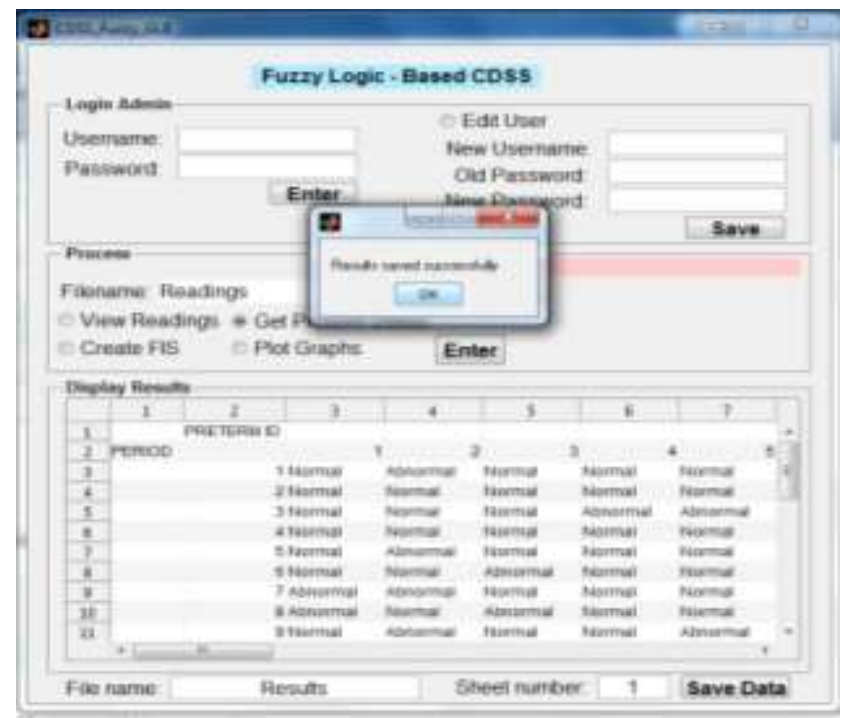

Fig. 12. Saving of the FIS Classification Results.

The predictions of the developed system for the thirty (30) neonates was taken 4 times daily (6:00am, 10:00am,2:00pm and 6:00pm) for seven (7) days, giving a total of twenty-eight (28) predictions per neonate as shown in Table II.

TABLE II. PREDICTIONS MADE FOR A NEONATE By THE DEVELOPED SYSTEM (FIS-CDSS)

\begin{tabular}{|l|l|}
\hline PERIOD & FIS-CDSS Prediction \\
\hline 1 & Normal \\
\hline 2 & Abnormal \\
\hline 3 & Normal \\
\hline 4 & Normal \\
\hline 5 & Normal \\
\hline 6 & Abnormal \\
\hline 7 & Normal \\
\hline 8 & Normal \\
\hline 9 & Normal \\
\hline 10 & Normal \\
\hline 11 & Normal \\
\hline 12 & Normal \\
\hline 13 & Normal \\
\hline 14 & Normal \\
\hline 15 & Normal \\
\hline 16 & Normal \\
\hline 17 & Abnormal \\
\hline 18 & Normal \\
\hline 19 & Normal \\
\hline 20 & Normal \\
\hline 21 & Normal \\
\hline 22 & Normal \\
\hline 23 & Normal \\
\hline 24 & Abnormal \\
\hline 25 & Abnormal \\
\hline 26 & Normal \\
\hline 27 & Normal \\
\hline 28 & Abnormal \\
\hline & \\
\hline
\end{tabular}




\section{CONCLUSION AND FURTHER WORK}

In this research, a CDSS based architecture for monitoring neonates in the NICU has been implemented. The developed system collects readings of the vital signs of neonates from measuring sensors attached to the wrist of the neonates. Fuzzy Inference System CDSS (FIS-CDSS) was developed for the three inputs: Temperature, Heart rate and Respiration rate (THR) based on their membership functions' value (low, medium, high) and twenty-seven (27) IF-THEN fuzzy rules using fuzzy logic toolbox. The FIS-CDSS maps the THR to an output status (Normal, Abnormal and Critical). The vital signs' readings were fed into the FIS-CDSS, which fuzzifies them and outputs the health status of the neonates.

The research work could be extended to measure or include more factors than the three basic vital signs temperature, heart beat rate and respiration. Other factors being observed by the specialist nurses such as transient clinical death, feeding rate and wavering weather could be included. The research work could also be extended to cover adults and other areas of health could be monitored and remotely reported to the physicians anywhere, anytime.

\section{REFERENCES}

[1] J.D. Marek and R.F. Roger, "Decision support systems" Decision systems laboratory, School of Information Sciences and Intelligent Systems Program University of Pittsburgh Pittsburgh, pp.3, 2002.

[2] M.M Abbasi and S. Kashiyarndi, "Clinical decision support systems: A discussion on different methodologies used in health care; Proceedings of the International Conference on Frontiers of Intelligent Systems, pp.1-15, 2020.

[3] J.A. Osheroff, J.M. Teich and B.F. Middleton, A roadmap for national action on clinical decision support. American Medical Informatics Association, 2006.

[4] C. Catley, and M. Frize, "A prototype XML-based implementation of an integrated intelligent neonatal intensive care unit" Research work published by University of Ottawa, Canada, 2003.

[5] K. Tan K, P.R.F. Dear and S.J. Newell, "Clinical decision support systems for neonatal care" published in The Cochrane Library, Issue 2; pp. 2-20, 2009.
[6] M. Prabhu, P.N. Senthil and K. Lakshmi, "Clinical decision support systems" Computer Sciences Corporation (CSC), pp. 1-19, 2014.

[7] J. Avansino and M.G. Leu, "Effects of CPOE on provider cognitive workload: a randomized crossover trial, pp. 547-552, 2012.

[8] M.J. Ball, J.V. Douglas, J. Lillis,"Health informatics: managing information to deliver value", PubMed indexed for MEDINE, Stud Health Technol Inform.;84(Pt1):305-8., 2001.

[9] J.A. Quinn: "Bayesian Condition Monitoring in Neonatal Intensive Care" $\mathrm{PhD}$ thesis submitted to Institute for Adaptive and Neural Computation School of Informatics University of Edinburgh, 2007.

[10] S. Nicklin, Y.A. Wickramasinghe and S.A. Spencer, "Neonatal intensive care monitoring current paediatrics"; Vol 14(1), pp. 1-7, 2004.

[11] STELLA Newsletter, "2nd International Workshop on. Flexible and Stretchable Electronics" Content Issue No. 6, 2010.

[12] L. Suresh, A.N. Latha A.N, R.B. Murthy, K.T. Alam, and J.K. Babu, Neonatal monitoring system Int. Journal of Engineering Research and Applications, ISSN : 2248-9622, Vol. 4(7), pp. 12-15, 2014.

[13] P.J. Pawar, B.V. Val, F. Bert-Jan and H. Hermens, "A framework for the comparison of mobile patient monitoring systems". Biomedical Journal, 2012.

[14] I.R. Okonkwo, B.I. Abhulimhen-Iyoha and A.A. Okolo, "Scope of neonatal care services in major Nigerian hospitals", Niger J Paed Vol 43(1), pp.8-13, 2016.

[15] A.A. Sobowale, S.O. Olabiyisi, and T.A. Abdul-Hameed, Development of a Framework for Computerized Health Management Information Systems in Nigeria: International Journal of Information and Communication Technology Research, 2011.

[16] Mednax Services, "Pediatrix Medical Group: For Parents, Your Baby and the NICU" Important Information from Your Health Care providers through The Centre for Research, Education and Quality. www.pediatrix.com/forparents , 2011.

[17] Ye Y. and Tong S. J A Knowledge-Based Variance Management System for Supporting the Implementation of Clinical Pathways.", Management and Service science, 2009, IEEE, pages 1 -4 (2009).

[18] Warren J, Beliakov G and Zwaag B. " Fuzzy logic in clinical practice Decision support system", Proceedings of the 33rd Hawaii International Conference on System Sciences. (2000).

[19] Prabhu M., Senthil P. N. and Lakshmi K., Clinical Decision Support Systems" Computer Sciences Corporation (CSC), pages 1-19 accessed in June, 2016. 